\title{
MULTIPLE SHELL PLANETARY NEBULAE: EVOLUTIONARY PATHS AND OBSERVED CONFIGURATIONS
}

\author{
LETIZIA STANGHELLINI \\ Osservatorio Astronomico di Bologna \\ via Zamboni, 33 I-40126 Bologna, Italy
}

\begin{abstract}
Recent observations of multiple shell planetary nebulae confirm the existing subdivision into two morphological types: attached halo and detached halo. The multiple shell phenomenon appears in about twentyfive percent of round and elliptical planetary nebulae of the Instituto de Astrofisica de Canarias morphological survey. The analysis of these nebulae together with that of their central stars lead to the interpretation that detached halo nebulae are formed through discontinuous mass loss at the Asymptotic Giant Branch tip. The attached halo nebulae, instead, are probably produced via dynamical nebular shaping.
\end{abstract}

\section{Introduction}

In the last two years a major observing program at the Instituto de Astrofisica de Canarias (IAC) has been carried out, aimed at the morphological study of northern galactic planetary nebulae (PNs) (Manchado et al., 1996). The survey includes images of 243 PNs through a choice of narrowband filters (see Manchado et al., this volume). There are 112 elliptical PNs (E) and 54 round PNs (R) in the IAC survey. Approximately twenty-five percent of the R and E PNs have shell multiplicity. This statistics should be integrate with the PNs observed by Balick (1987), which were excluded $a$ priori from the IAC survey. The IAC survey provide a wide database for the study of the origins of shell multiplicity in galactic PNs. In order to understand the phenomenon of multiple shell formation, and to relate it with the evolution of the central stars and to the nebular dynamics, we also need a large database of the physical parameters of the central stars, and accurate distances and expansion velocities of the nebulae. Early studies on multiple shell planetary nebulae (MSPN) have been proposed by Stanghellini 


\section{Stanghellini}

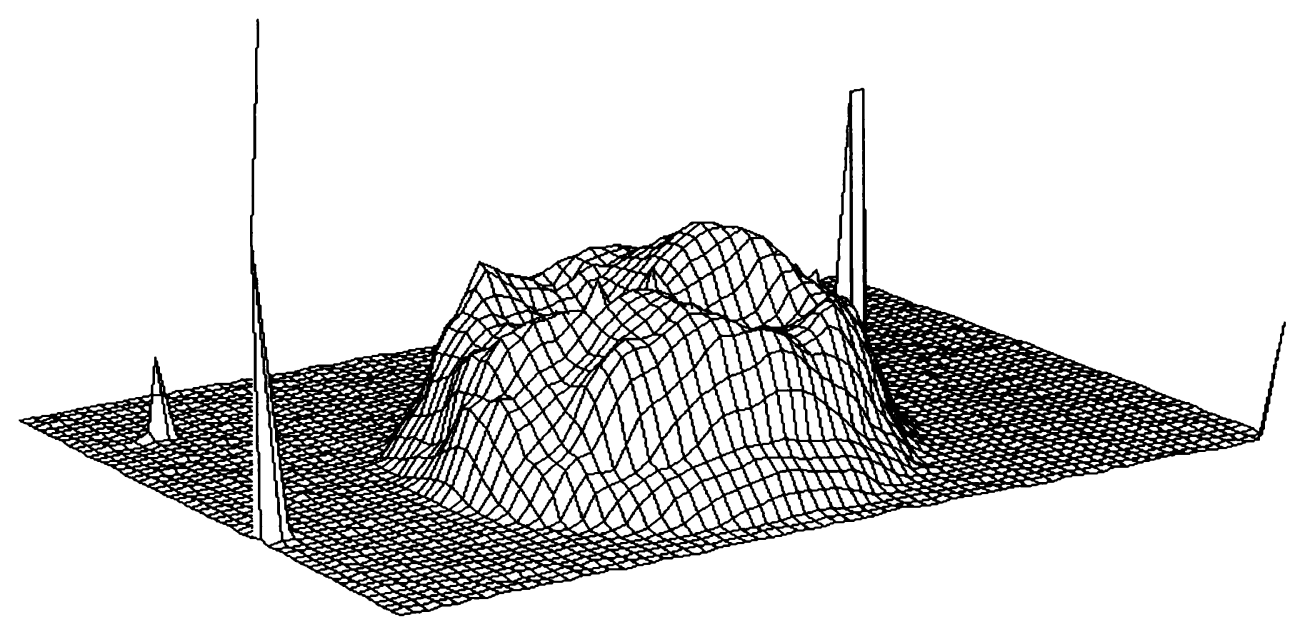

Figure 1. Surface brightness of NGC 3587 (attached halo PN).

\& Pasquali (1995), who completed a limited analysis of central stars correlated to the MSPN observed by Schwarz et al. (1992). The results were encouraging, showing that a lot of insight can be gained by correlating the properties of stars and nebulae within a special morphological class. Guerrero et al. (1996) have done a similar study in the case of M1-46, where the accurate spectral information for both nebula and star support the interpretation that this PN has been formed by discontinuous shell ejection. We feel that it is important to continue our general analysis of MSPN and their central stars with the new IAC data. So far, we have found reliable stellar parameters only for a handful of objects, and we plan to expand the stellar database of northern PNs by acquiring suitable information on those central stars whose PNs show multiple shell morphology.

\section{Detailed morphological analysis of multiple shell planetary nebulae}

The MSPN of both northern (Manchado et al., 1996) and southern (Schwarz et al., 1992) samples can be sorted remarkably well into two main classes: the attached halo (AH) and detached halo (DH) PNs. For homogeneity with previous work we use the definitions given originally by Chu et al. 1987, also used by Stanghellini \& Pasquali 1995. The attached halo PNs (an example in Fig. 1) are characterized by a continuous brightness decrease from inside out the shells, while the detached halo PNs (Fig. 2) show a minimum brightness between the inner and the outer shells. It is worth noting that several authors call "double shell" the AH PNs, and "halo PNs" the DH PNs. A second-order difference between the northern and 


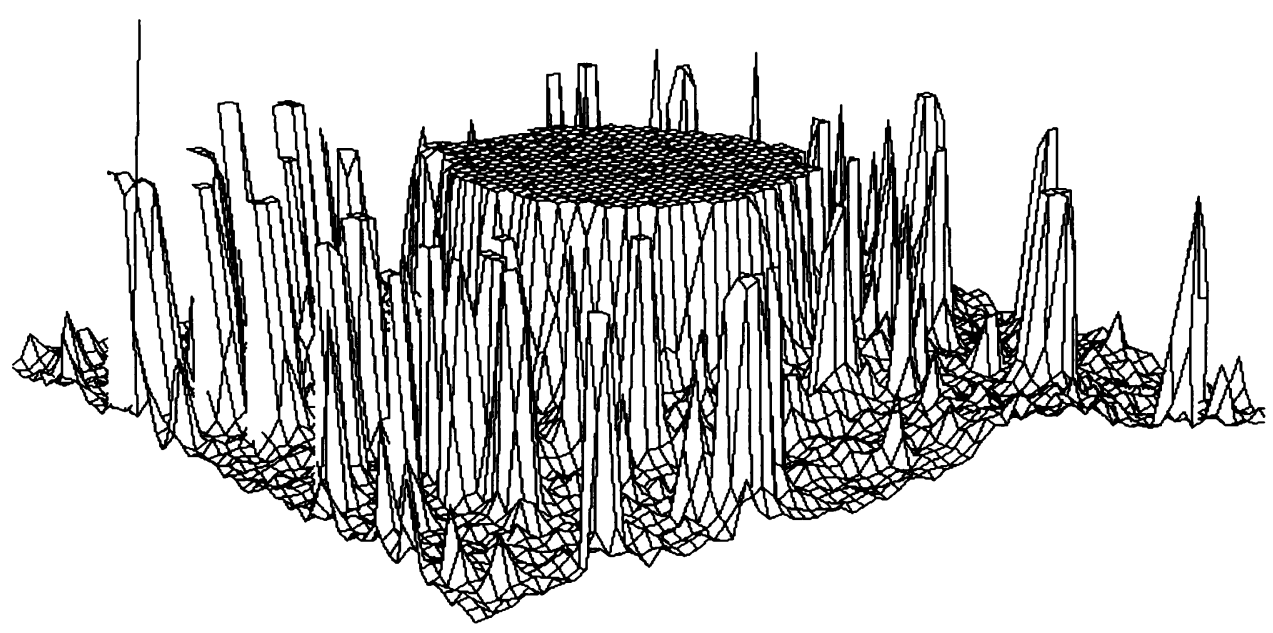

Figure 2. Surface brightness of NGC 2438 (detached halo PN).

southern samples is that, on the Manchado et al. sample we did not see a sharp ellipticity discrimination between $\mathrm{AH}$ and DH PNs, as the one shown by Stanghellini \& Pasquali on the Schwarz et al. sample.

\section{Central star connection}

As mentioned in the introduction, only for a fraction of central stars in the Manchado et al. set do we know the fundamental parameters, available in the literature. We thus perform our limited analysis on these objects. We derive the stellar temperatures with a Zanstra analysis, while the stellar luminosities are obtained from the statistical distances (Cahn et al., 1992) and the stellar magnitudes (Acker et al., 1992). We then place the central stars of these MSPN on the HR diagram, to study their evolution in correlation to the morphology of the shells. In Figure 3 we show the resulting loci of the central stars on the HR plane, respectively with filled symbols for $\mathrm{AH}$ and with open symbols for DH PNs. The stellar tracks are synthetic post-asymptotic giant branch (AGB) calculations by Stanghellini \& Renzini (1993), corresponding to $0.55,0.7$, and $1.4 \mathrm{M}_{\odot}$. The errorbars on the temperatures and luminosities reflect the propagation of all observational errors, but do not include the uncertainties for the statistical distances.

Figure 3 shows a segregation between the two group of objects: it is evident that the attached halo PNs host stars that cluster around smaller mass tracks. If we include in the sample the stars analyzed by Stanghellini \& Pasquali (1995), we obtain that the mean mass of the AH central stars, averaged over 24 objects, is $\left\langle M>_{\mathrm{AH}}=0.64 \mathrm{M}_{\odot}\right.$, while the mean mass for $\mathrm{DH}$ central stars, over 16 objects, is $0.72 \mathrm{M}_{\odot}$. We note from Figure 3 


\section{Stanghellini}

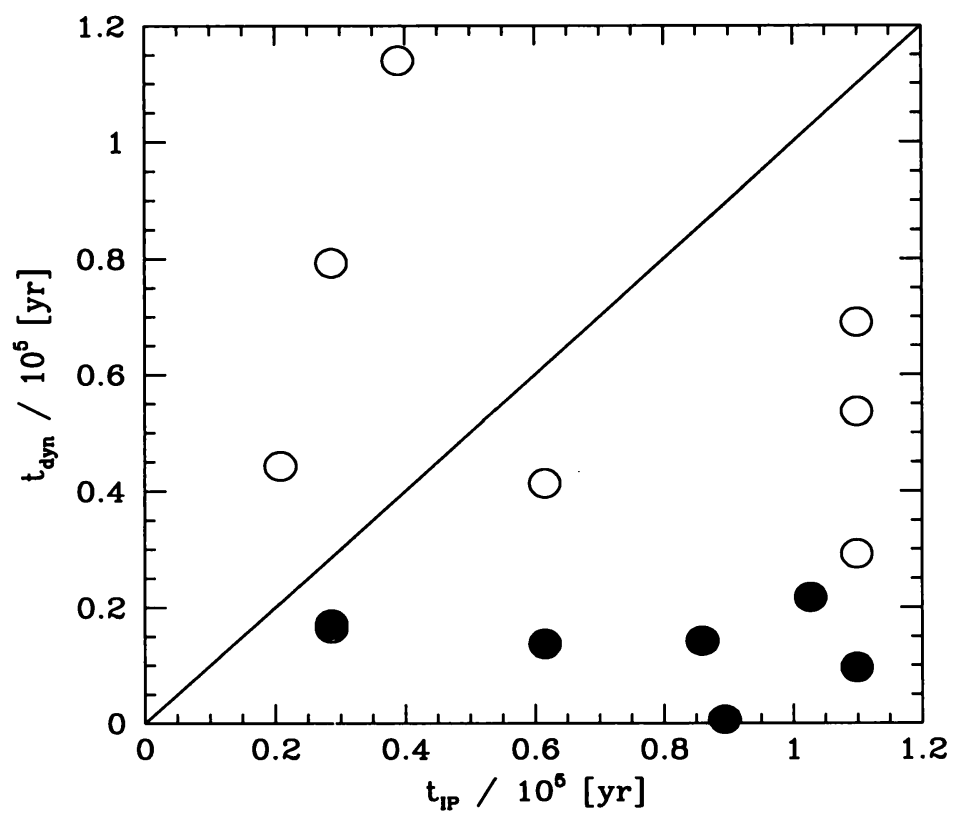

Figure 3. Central stars of attached (filled symbols) and detached (open symbols) MSPN on the HR diagram.

that AH PNs have central stars with $\log \mathrm{L}<2 \mathrm{~L}_{\odot}$. If this results from a selection effect, we should calculate the average mass of stars hosted by AH PNs above that limit, to have homogeneity with the DH sample. In this way, We obtain for $\mathrm{AH}$ central stars that $\left\langle M>_{\mathrm{AH}}=0.61 \mathrm{M}_{\odot}\right.$. Thus average stellar masses are typical of galactic PNs for AH PNs, while for DH PNs the masses are remarkably larger. It seems clear from Figure 3 that $\mathrm{AH}$ PNs do not evolve into DH PNs, nor vice versa.

\section{Shell formation}

The multiple shell PNs may have different origins. The possible mechanisms of formation of multiple shell are: (1) differential (i. e. "gasping") mass loss at the AGB tip; (2) differential mass loss during the PN lifetime, with the possible production of a "born again" $\mathrm{PN}$; (3) dynamical shell evolution, when the hot stellar wind shocks, then forms a hot bubble, which later compresses the outer shell and produces an outer, fainter shell. A review on this subject, and a list of the main papers related to it, can be found in Stanghellini \& Pasquali (1995). These different evolutionary paths have different observable consequences. In case (1) the dynamical intershell time is similar to the time interval between successive ejection that form independent shells (interpulse time). In case (2) the dynamical time of the outer 


\section{MULTIPLE SHELL PLANETARY NEBULAE}

shell should be at least comparable to the stellar evolutionary time to the born again configuration. In case (3) the interpulse time is larger than the dynamical intershell time, while the ionized mass of the outer shell should increase with time.

We verify these observables on the IAC sample with the same procedure used by Stanghellini \& Pasquali. First, we derive the location of the central star on the HR diagram, so that we can infer the stellar masses. Then we calculate the interpulse time at the AGB, from Vassiliadis \& Wood (1993, 1994) and we compare this time scale to the dynamical intershell time, for the two types of MSPN. In Figure 4 we show the results of our limited and preliminary analysis. The scatter is very large and the errors can add up to a horizontal errorbar of $0.3 \times 10^{5}$ years. In this Figure, the open circles in the right side are upper limits to the interpulse time. Even with the large scatter, we basically obtained the same qualitative results than those by Stanghellini \& Pasquali (1995), thus confirming that gasping mass loss evolution may occur only to progenitors of DH PNs. It is worth noting that all distances used here are statistical distances (Cahn et al., 1992), whose errors can be up to fifty percent. Another important source of errors are the expansion velocities, from Acker et al. (1992). To obtain better measurements on these velocities we are actively observing spectroscopically all the MSPN in the IAC survey, and the results concerning the nebular morphology-stellar evolution connection will be soon in press.

\section{Conclusions}

As a continuing investigation on the origins of the observed planetary nebula shapes, we have studied the northern galactic MSPN and their connections to stellar evolution. We preliminary confirm the results, found in previous studies (Stanghellini \& Pasquali, 1995), that the attached halo morphology originates from dynamical evolution, while the detached halo morphology may originate from gasping mass loss at the AGB thermally pulsating cycle. Our aim for the future is to study the expansion velocities in detail, in order to obtain reliable dynamical times for all MSPN in the IAC sample.

\section{Acknowledgements}

This work would have not been possible without the contributions of Arturo Manchado and Martin A. Guerrero, whom I warmly thank. I acknowledge the hospitality of the IAC and the STScI, where, respectively, this work was developed and written. 


\section{Stanghellini}

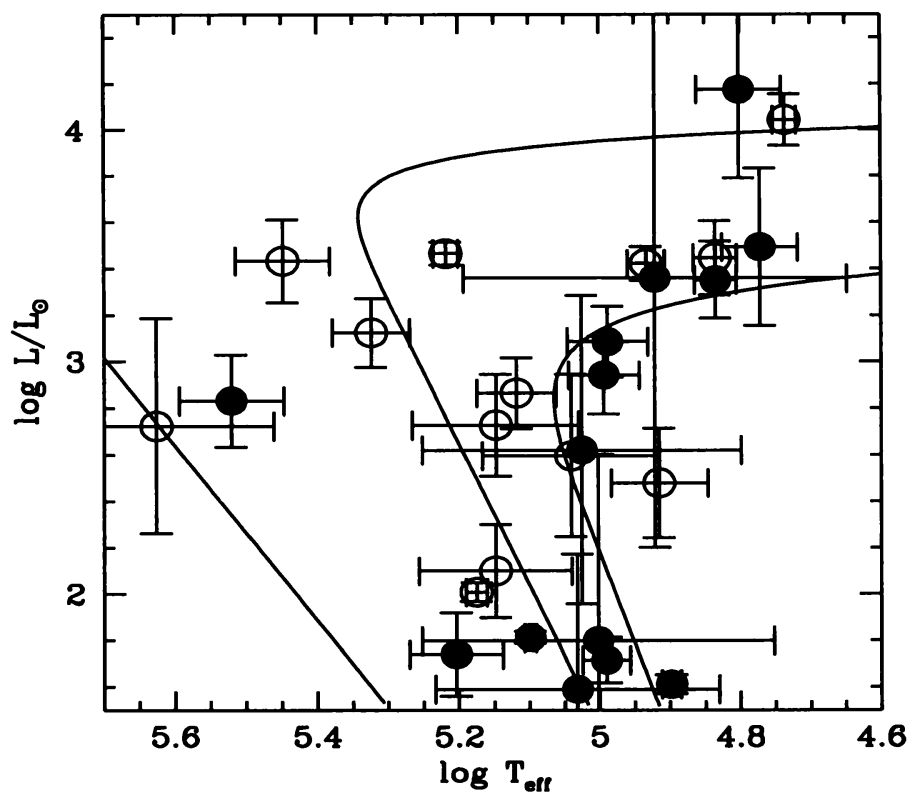

Figure 4. dynamical intershell times versus interpulse (AGB) evolutionary times for attached (filled symbols) and detached (open symbols) MSPN. Given the large uncertainties in the expansion velocities, the results to be drawn from this plot should be considered preliminary.

\section{References}

Acker, A., Ochsenbein, F., Stenholm, B., Tylenda, R., Marcout, J., \& Schohn, C. 1992, Strasbourg-ESO Catalogue of Galactic planetary nebulae (Garching: ESO)

Balick, B. 1987, AJ 94, 671

Cahn, J. H., Kaler, J. B., \& Stanghellini, L. 1992, A\&AS 94, 399

Chu, Y-H., Jacoby, G. H., \& Arendt, R. 1987, ApJS 64, 529

Guerrero, M. A., Manchado, A., Stanghellini, L., \& Herrero, A. 1996, ApJ 464, 847

Manchado, A., Guerrero, M., Stanghellini, L., \& Serra-Ricart, M. 1996, The IAC Morphological Catalog of Northern Galactic planetary nebulae (Tenerife: IAC)

Schwarz, H. E., Corradi, R., \& Melnick, J. 1992, A\&AS 96, 23

Stanghellini, L., \& Pasquali, A. 1995, ApJ 452, 286

Stanghellini, L, \& Renzini, A. 1993, in planetary nebulae, ed. A. Acker \& R. Weinberger (Dordrecht: Kluwer), 473

Vassiliadis, E., \& Wood, P. 1993, ApJ 413, 641

Vassiliadis, E., \& Wood, P. 1994, ApJS 92, 125 